International Journal of Advances in Chemistry (IJAC) Vol.2,No.1 February 2016

\title{
A HPLC-UV METHOD FOR DETEERMINATION OF THREE PESTICIDES IN WATER
}

\author{
Fuad Al-Rimawi \\ Faculty of Science and Technology, Al-Quds University, P.O. Box 20002, East \\ Jerusalem, Palestinian Authority
}

\begin{abstract}
HPLC method is developed and validated for determination of three pesticides (abamectin, imidacloprid, and $\beta$-cyfluthrin) in water. These pesticides are used widely in agriculture for crops protection, and may be leached to the groundwater. Reversed-phase method with C18 column $(5 \mu \mathrm{m}, 250 \mathrm{~mm} \times 4.6 \mathrm{~mm}$ inner diameter) using a mobile phase consisting of acetonitrile/water (v:v = 4:1) at a flow rate of $1.5 \mathrm{~mL} / \mathrm{min}$ and UV detection at $220 \mathrm{~nm}$ was used. This method is validated according to new methods which include accuracy, precision, linearity and range, limit of detection and limit of quantitation. The current method exhibits good linearity over the range of 1-1000 ppb for abamectin, 0.5-1000 ppb for imidacloprid, and 0.4-1000 ppb for $\beta$-cyfluthrin with $r^{2}$ greater than 0.990. The percentage recovery of the method at three concentration levels $(5,100$, and $1000 \mathrm{ppb})$ is within 97.6 to $101.5 \%$ for the three pesticides. Relative standard deviation of the area of six replicate injections of each pesticide at three concentration levels (5.0, 100.0, and $1000.0 \mathrm{ppb}$ ) was found to be less than 1\% which reflect the precision of the method. Limit of quantitation of the three pesticides using this method is low (1.0, 0.5, and 0.4 ppb) for abamectin, imidacloprid, and $\beta$-cyfluthrin, respectively which enables the determination of these three pesticides in water at low concentration levels.
\end{abstract}

Keywords : Abamectin, Imidacloprid, $\beta$-cyfluthrin, pesticides, HPLC-UV, validation

\section{INTRODUCTION}

Pesticides are applied to crops to increase their yield. However, pesticide residues in different environmental compartments e.g. soil, water, fruits, and vegetables have adverse effects on the human health. In this respect, continuous monitoring of pesticides in different environmental matrices at low concentration levels is required. HPLC-UV is a simple and robust method for determination of pesticides. Therefore, the objective of this study is to develop and validate a method for simultaneous determination of three pesticides (abamectin, imidacloprid, and $\beta$ cyfluothrin, their structures are shown in Figure 1) in water. These three pesticides are used for crops protection in large quantities. Abamectin is an insecticide belongs to the family of avermectins which are macrocyclic lactones. Although, abamectin has a strong tendency to bind to soil [1], it can reach the groundwater through rain or through sandy soil. Imidacloprid is stable in the soil for several months, but can reach groundwater during water runoff [2]. $\beta$-cyfluthrin pesticide has low solubility in water and has strong tendency to adsorb to soil [3]. These pesticides present in water with low concentrations, a method therefore with low limit of detection (LOD) and limit of quantitation (LOQ) is required to detect these pesticides at low concentrations.

Abamectin has been analyzed by HPLC with direct UV detection [4, 5], mass spectrometry (MS) $[6,7]$, and fluorescence detection [8, 9]. Several methods have been reported for the determination of imidacloprid in different environmental matrices by HPLC with UV-detection [10-12], and with mass spectrometry [13]. The determination of B-cyfluthrin in different environmental matrices was also reported by means of HPLC with UV-detection [14-15] and chemiluminescence [16]. However, no method for determination of these three pesticides together was reported. A method for simultaneous determination in solusions containing these three pesticides is presented in this work. The method for analysis of samples with these three pesticides in the current work is simple, where HPLC technique is employed after liquid-liquid extraction of the pesticides from water samples. Furthermore, UV detector was employed for the detection of the pesticides. 
International Journal of Advances in Chemistry (IJAC) Vol.2,No.1 February 2016<smiles>O=[N+]([O-])NC1=NCCN1Cc1ccc(Cl)nc1</smiles>

(1)

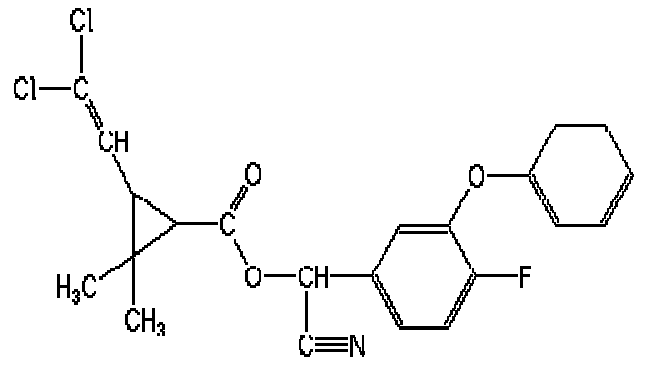

(2)

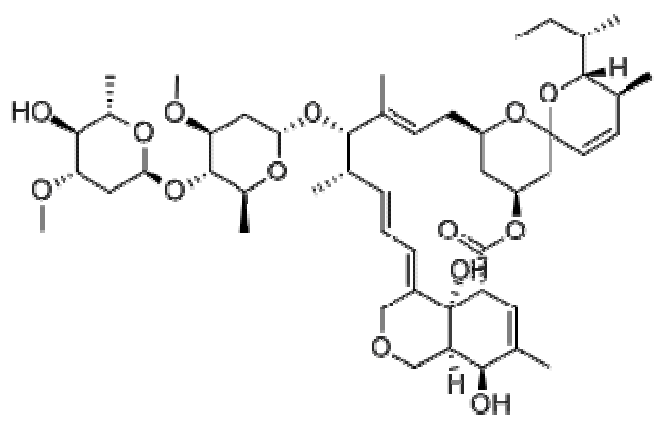

(3)

Fig 1: Structures of the three pesticides analyzed in this study: (1) imidacloprid (2) $\beta$-cyfluthrin (3) abamectin.

\section{Materials and methods}

\section{1. Chemicals}

Acetonitrile (HPLC grade), n-hexane 95\% (HPLC grade), dichloromethane 99.5\% (HPLC grade), phosphoric acid (85\%), and isopropyl alcohol are from Merck (Darmstadt, Germany). Abamectin (avermectin B1b, 99\%, HPLC grade), $\beta$-Cyfluthrin ((RS)-a-cyano-4-fluoro-3phenoxybenzyl(1RS,3RS)-3-(2,2dichllorovinyl)-2,2dimethylcyclopropane-carboxylate, $\quad 99.8 \%$, HPLC grade), and Imidacloprid (1-[(6-chloropyridin-3-yl) methyl]-N-nitro-4, 5-dihydroimidazol2-amine, 99.9\%, HPLC grade), were obtained from Sigma-Aldrich. 


\section{2 Apparatus}

HPLC system (Merck Hitachi Lachrom Elite HPLC system, Japan) with a pump, an autosampler, column oven, and a UV-detector was employed. The chromatographic column C18 $(25 \mathrm{~cm}$ length, $4.6 \mathrm{~mm}$ inner diameter, and $4.0 \mu \mathrm{m}$ particles) is from Waters Corporation (Milford, Massachusetts, USA).

\subsection{Extraction}

$100.0 \mathrm{ml}$ of water was extracted with $3 \times 60 \mathrm{ml}$ of organic solvent: $\mathrm{n}$-hexane (for Imidacloprid and $\beta$-Cyfluthrin) and dichloromethane (for Abamectin). The organic extracts were then collected and concentrated with rotary vacuum evaporation until few drops of solution was left. Then, $1.0 \mathrm{ml}$ of acetonitrile was added. After that, the sample is ready for analysis by HPLC.

\subsection{Standard solution preparation}

\subsubsection{Standard solutions and stabdard curve plotting}

Stock standard solution of each pesticide $(1000 \mathrm{ppb})$ was prepared in acetonitrile. Different concentrations of each pesticide were then prepared from the stock solution by dilution using mobile phase as diluent. The following diluted concentrations were prepared: 1.0, 10.0, 100.0, $300,500.0,700.0$, and $1000.0 \mathrm{ppb}$ (for abamectin), 0.5, 10.0, 100.0, 300, 500.0, 700.0, and $1000.0 \mathrm{ppb}$ (for imidacloprid), 0.4, 10.0, 100.0, 300, 500.0, 700.0, and $1000.0 \mathrm{ppb}$ (for $\beta$ cyfluthrin). Each of these solutions was injected into the HPLC and peak areas were recorded and plotted versus the concentration of the pesticide.

Please give the equations for the stabdard curves.

\subsubsection{Percentage recovery (Accuracy) of the method}

Percentage recovery of the current method for determination of the three pesticides was studied by spiking each pesticide in distilled water that contains no pesticides, at three concentration levels $(5.0,100.0$, and $1000.0 \mathrm{ppb})$. Then the spiked samples were extracted using the same procedure followed in the extraction of groundwater samples (see section 2.3), and analyzed using the same method by HPLC-UV.

\subsubsection{Precision of the method}

The precision of the method was evaluated by calculating relative standard deviation (RSD) of the areas of six replicate injections of each pesticide at the three concentration levels $(5.0,100.0$, and $1000.0 \mathrm{ppb}$ ).

\subsubsection{Limit of detection (LOD) and limit of quantitation (LOQ)}

LOD of the pesticides was calculated by preparing different solutions with low concentrations that is expected to produce a response that is 3-10 times baseline noise. The solutions are injected and the signal to noise ratio $(\mathrm{S} / \mathrm{N})$ are recorded. LOD is selected as the concentration of pesticide that gives a $\mathrm{S} / \mathrm{N}$ ratio of 3-10. LOQ is determined in the same manner and selected as the concentration of pesticide that gives an $\mathrm{S} / \mathrm{N}$ ratio of 10-20. 


\section{Results and discussion}

\subsection{Method development}

Preliminary studies involved trying C8 and C18 reversed-phase columns and testing several mobile phase compositions and using different chromatographic parameters for the separation of the three studied pesticides. A C18 column $(25 \mathrm{~cm}$ length, $4.6 \mathrm{~mm}$ inner diameter, and $4.0 \mu \mathrm{m}$ particles) was used as a stationary phase for separation. As a mobile phase, a mixture of water and acetonitrile was used ( $80 \%$ acetonitrile and $20 \%$ water, v/v). Isocratic elution was performed for analysis using a flow rate of $1.5 \mathrm{ml} / \mathrm{min}$, and UV detection at a wavelength of $220 \mathrm{~nm}$. Injection volume was set to be $20 \mu \mathrm{L}$ for all samples and standards. Figure 2 shows chromatogram of abamectin, imidacloprid, and $\beta$-cyfluthrin separated using the current developed method.

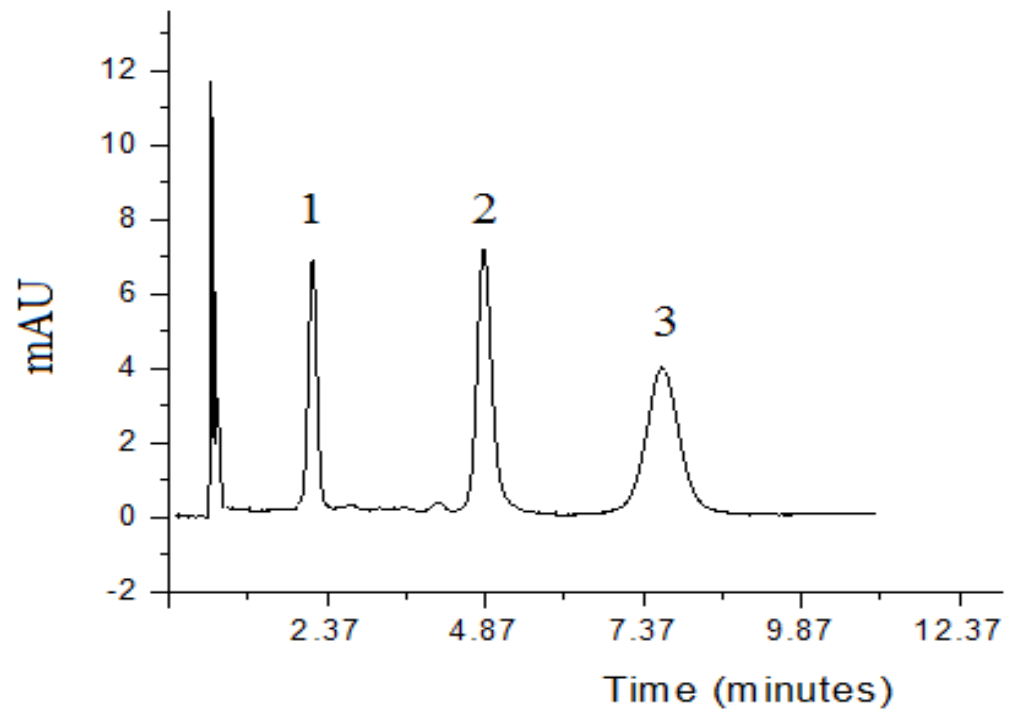

Figure 2: Chromatogram of the three pesticides analyzed in this study. (1) imidacloprid, (2) $\beta$-cyfluthrin,

(3) abamectin.

\subsection{Method validation}

\subsubsection{Linearity and range}

The correlation coefficient $\left(\mathrm{r}^{2}\right)$ of the calibration curve should not be less than 0.990 for the least squares method of analysis [17]. To evaluate linearity of the method, different calibration standards of the pesticides were analyzed by HPLC-UV and the responses are recorded. A plot of the peak areas of the pesticides versus concentration (in ppb) was found to be linear in the range of 1-1000 ppb for abamectin, 0.5-1000 ppb for imidacloprid, 0.4-1000 ppb for ß-cyfluthrin with $\mathrm{r}^{2}$ greater than 0.990. This result indicates that these three pesticides can be determined in groundwater at wide concentration range.

\section{2. 2 Recovery (accuracy)}

The accuracy of an analytical method measures the closeness between true value and value found (i.e., accuracy is a measure of exactness of an analytical method). Accuracy is measured as the percent of analyte recovered after spiking samples in a blank. To test the accuracy, a minimum of nine determinations over a minimum of three concentration levels covering the specified range 
International Journal of Advances in Chemistry (IJAC) Vol.2,No.1 February 2016

are needed. It is performed at three concentrations covering the range of the method. At each level studied, replicate samples are evaluated. The RSD of the replicates provides the analysis variation and gives an indication of the precision of the test method. The mean of the replicates, expressed as \% of label claim, indicates the accuracy of the test method. The mean recovery of the assay should be within $100 \pm 5.0 \%$ at each concentration over the studied range [18].

For determination of the recovery of the investigated pesticides, these pesticides are spiked in distilled water followed by liquid-liquid extraction, and analysis by HPLC-UV. The average recovery for each level has been calculated by proportion of the area of the peak of pesticide resulted from the spiked solution to the area of the peak of that pesticide resulted from a standard solution prepared from standard of that pesticide. Results have showed that the current method has good recovery (from 97.6 to $101.5 \%$ ) for the three pesticides at the three concentration levels studied $(5.0,100.0$, and $1000.0 \mathrm{ppb}$ ), and with a relative standard deviation lower than $1.0 \%$ (see Table 1).

Table 1: Recovery of abamaectin, imidacloprid, and $\beta$-cyfluthrin at three concentration levels $(5,100$, and

\begin{tabular}{|c|c|c|c|}
\hline \multirow{4}{*}{ Pesticide } & \multicolumn{3}{|c|}{$1000 \mathrm{ppb})$} \\
\hline & \multicolumn{3}{|c|}{ Recovery / \% } \\
\hline & \multicolumn{3}{|c|}{ Concentration (ppb) } \\
\hline & 5.0 & 100.0 & 1000.0 \\
\hline \multirow{4}{*}{ Abamaectin } & $101.0,100.7,100.9$ & $98.2,99.5,100.0$ & $99.3,98.1,97.6$ \\
\hline & Mean: 100.9 & Mean: 99.2 & Mean: 98.3 \\
\hline & SD: 0.15 & SD: 0.93 & SD: 0.87 \\
\hline & RSD: 0.15 & RSD: $0.94 \%$ & RSD: 0.88 \\
\hline \multirow{4}{*}{ Imidacloprid } & $101.0,101.5,100.8$ & $100.3,99.7,99.1$ & $99.2,100.5,98.9$ \\
\hline & Mean: 101.1 & Mean: 99.7 & Mean: 99.5 \\
\hline & SD: 0.36 & SD: 0.60 & SD: 0.85 \\
\hline & RSD: $0.36 \%$ & RSD: $0.60 \%$ & RSD: $0.85 \%$ \\
\hline \multirow{4}{*}{$\beta$-cyfluothrin } & $100.5,99.8,100.0$ & $98.7,97.8,99.1$ & $100.5,100.0,99.8$ \\
\hline & Mean: 100.1 & Mean: 98.5 & Mean: 100.1 \\
\hline & SD: 0.36 & SD: 0.67 & SD: 0.36 \\
\hline & RSD: $0.36 \%$ & RSD: $0.68 \%$ & RSD: $0.36 \%$ \\
\hline
\end{tabular}

*SD: standard deviation.

**RSD: relative standard deviation. 


\subsubsection{Precision}

Precision is the measure of the repeatability of a method under normal operation and is normally expressed as the RSD for a number of samples. There are two types of precision: repeatability and intermediate precision (ruggedness).

\subsubsection{Repeatability}

Repeatability is the closeness between independent test results obtained with the same method on identical test material in the same laboratory by the same operator using the same equipment. It is determined from a minimum of nine determinations covering the specified range of the procedure. RSD for replicate injections should not be greater than $1.5 \%$ [19].

Repeatability of the current method for determination of the three pesticides was evaluated by calculating the RSD of the peak areas of six replicate injections of standard solutions with three concentrations $(5.0,100.0$, and $1000.0 \mathrm{ppb}$ ), which was found to be less than $1.0 \%$ (data not shown). These results show that the current method for determination of the pesticides is repeatable.

\section{2.3.2 Intermediate precision (Ruggedness)}

Ruggedness of a method measures the repeatability of the result obtained with the same method, on the same sample, in the same laboratory, but by different operators and in different day. Intermediate precision of the method was evaluated by calculating the $\%$ recovery of the pesticides at three concentration levels $(5.0,100.0$, and $1000.0 \mathrm{ppb})$ by another analyst in different day. Results of this study showed that the \% recovery obtained by the second analyst is comparable to that obtained by the main analyst and ranges from 97.2 to $101.9 \%$ (data not shown), indicating that this method is rugged.

\subsection{Selectivity}

Selectivity is the ability to assess the analyte in the presence of other analytes and other components that may be present in the matrix or sample [20]. It is a measure of interferences from such components, ensuring that a response is due to a single component only. Selectivity of the current method was demonstrated by good separation of the pesticides from each other with good resolution (resolution between imidacloprid and $\beta$-cyfluthrin is 3.5 , and the resolution between $\beta$-cyfluthrin and abamectin is 2.1).

\subsubsection{Limit of detection (LOD) and limit of quantitation (LOQ)}

LOD is the lowest concentration of analyte in a sample that can be detected but not necessarily quantitated, and can be determined by preparing a solution that is expected to produce a response of about 3 to 10 times base line noise. The solution is injected three times, and the signal to noise ratio for each injection are recorded. The concentration of the solution is considered as a LOD if the $\mathrm{S} / \mathrm{N}$ ratio is between 3 and 10 . LOQ can be determined in the same manner but with $\mathrm{S} / \mathrm{N}$ ratio of $10-20$.

LOD and LOQ of the three pesticides using the current method in this study were found to be low (see Table 2) which enables the detection and quantitation of theses pesticides in ground water at low concentration levels. 
International Journal of Advances in Chemistry (IJAC) Vol.2,No.1 February 2016

Table 2: LOD and LOQ of abamectin, imidacloprid, and $\beta$-cyfluthrin using the method employed in this study.

\begin{tabular}{ccc} 
Pesticide & LOD (in ppb) & LOQ (in ppb) \\
\hline Abamaectin & 0.3 & 1.0 \\
Imidacloprid & 0.1 & 0.5 \\
$\beta$-cyfluothrin & 0.1 & 0.4 \\
\hline
\end{tabular}

\subsection{Application of the method to real water samples}

After successful development of this method for determination of the three pesticides in water, it was employed for their analysis in groundwater of West Bank/Palestine. Twenty five water samples were collected from ground water boreholes in the lower Jordan Valley/Jericho District and analyzed for the three pesticides by this HPLC method. Results showed that the concentration of abamectin, imidacloprid, and $B$-cyfluthrin ranges between $1.24 \mathrm{ppb}-81.71 \mathrm{ppb}, 1.60 \mathrm{ppb}-$ $325.00 \mathrm{ppb}$, and $1.10-24.46 \mathrm{ppb}$, respectively. It is concluded that this HPLC-UV method can be used for quantitative determination of these three pesticides in groundwater.

\section{CONCLUSIONS}

A simple, accurate, precise, and selective HPLC method has been developed and validated for determination of three pesticides ( $\beta$-cyfluthrin, abamectin, and imidacloprid) in groundwater. The method is curate in determination of these pesticides with a wide dynamic range (1-1000 ppb for abamectin, 0.5-1000 ppb for imidacloprid, 0.4-1000 ppb for B-cyfluthrin) with recovery from 97.6 to $101.5 \%$. Low LOD and LOQ of the pesticides analyzed in this study enable the detection and quantitation of them in water at low concentrations. This validated method can be employed for the determination of these pesticides in real water samples, including groundwater, surfacewater and wastewater.

\section{References}

[1] J. Frank \& H. Lawrence, (1999), Multiresidue Determination of Abamectin, Doramectin, Ivermectin, and Moxidectin in Milk Using Liquid Chromatography and Fluorescence Detection. Journal of AOAC International, Vol. 82, No. 6, pp 1340-1344.

[2] W. Zheng, \& W. Liu, (1999) Kinetics and Mechanism of the Hydrolysis of Imidacloprid". Pesticides Science, Vol. 55, No 4, pp 482-485.

[3] C. Anderson, (1986) Degradation behavior of 14C-Cyfluthrin in natural water. Mobay Chemical Corp. Report No. 93044.

[4] J. Li, \& C. Qian, (1996) "Determination of avermectin B1 in biological samples by immunoaffinity column cleanup and liquid chromatography with UV detection", J. AOAC Int. Vol. 79, No. 5, pp 1062-1067.

[5] A. Valenzuela, D. S. Popa, M. J. Redondo, \& J. Manes, (2001) Comparison of various liquid chromatographic methods for the analysis of avermectin residues in citrus fruits. Journal of Chromatography A. Vol. 918, pp 59-65.

[6] K. Yoshii, A. Kaihara, Y. Tsumura, S. Ishimitsu, \& Y. Tonogai, (2000). Liquid chromatographic determination of emamectin, milbemectin, ivermectin and abamectin in crops and confirmation by liquid chromatography-mass spectrometry, J. Chromatogr. A Vol. 896, No. 1-2, pp 75-85. 
International Journal of Advances in Chemistry (IJAC) Vol.2,No.1 February 2016

[7] A. I. Valenzuela, M. J. Redondo, Y. Pico, \& G. Font, (2000) Determination of abamectin in citrus fruits by liquid chromatography-electrospray ionization mass spectrometry. J. Chromatogr. A Vol. 871, pp 57-65.

[8] S. V. Prabhu, R.J. Varsolona, T.A. Wehner, R.S. Egan, \& P.C. Tway, (1992) Rapid and Sensitive HPLC-Method for the Quantitation of Abamectin and its Delta 8,9-Isomer. J. Agr. Food Chem. Vol. 40 No. 4, pp 622-925.

[9] J. A. Cobin, \& N.A. Johnson, (1996). Liquid chromatographic method for determination of total avermectin B1 and 8,9-Z-avermectin B1 residues in hops. J. AOAC Int. Vol. 79, No. 2, pp 503-507.

[10] S. Baskaran, R.S. Kookana, \& R. Naidu, (1997) Determination of the insecticide imidacloprid in water and soil using high-performance liquid chromatography, Journal of Chromatography A, Vol. 787, No. 1-2, pp 271-275.

[11] P. Samnani, K.. Vishwakarma, \& S.Y. Pandey (2011) Simple and sensitive method for determination of imidacloprid residue in soil and water by HPLC, Bull Environ Contam Toxicol. Vol 86, No. 5, pp 554-558.

[12] H. Obana, M. Okihashi, K. Akutsu, Y. Kitagawa, \& S. Hori, (2002) Determination of Acetamiprid, Imidacloprid, and Nitenpyram Residues in Vegetables and Fruits by High-Performance Liquid Chromatography with Diode-Array Detection, J Agric Food Chem. Vol. 50 No. 16, pp 4464-4467.

[13] R. Schoening, \& R. Schmuck, (2003) Analytical determination of imidacloprid and relevant metabolite residues by LC MS/MS, Bulletin of Insectology Vol. 56, No. 1, pp 41-50.

[14] L. Vodeb, \& B. Petanovska-Ilievska, (2006) High speed determination of $\beta$-cyfluthrin in EC and SC pesticide formulations by reverse phase and normal phase liquid chromatography, J. Liquid chromatography \& Related Techniques, Vol. 29, No. 5, pp 649-660.

[15] L. Vodeb, \& B. Petanovska-Ilievska, (2006) HPLC-DAD with different types of column for determination of $\beta$-cyfluthrin in pesticide formulations, Acta Chromatographica, Vol. 17, pp 88-201.

[16] M.M. Galera, .M.D. Garcia, \& R.S. Valverde (2006) Determination of nine pyrethroid insecticides by high-performance liquid chromatography with post-column photoderivatization and detection based on acetonitrile chemiluminescence. J Chromatogr A. Vol. 1113, No. 1-2, pp 191-197.

[17] International Conference on Harmonization (ICH), "Validation of Analytica 